over the country, Hacettepe University Medical Faculty Obstetrics and Gynecology Department, Obstetrics Unit, in October 2009, just before pandemic influenza vaccine is implemented clinically to evaluate the perception of vaccine. Our study was conducted as applying questionaire to 86 pregnant women admitting to Hacettepe University Medical Faculty Obstetrics and Gynecology Department, Obstetrics Unit. Age, trimestr and gravida of pregnancy, seasonal influenza vaccine status, whether she finds pandemic influenza vaccine necessary was noted after questionnaire. Pregnant women contributing to our questionaire were between 18-39 years of age, 13 of them were in first trimestr, 31 were in second trimestr, 42 were in third trimestr. Out of 86 pregnant women 66 expresses that they did not find it necessary to have pandemic influenza vaccine. In our study it was found that pregnant women find pandemic influenza vaccine necessary with a percentage of $23.3 \%$ and although the study population is small, finding it necessary was not effected by mother's age, trimestr of pregnancy, gravida of pregnancy. The only factor demonstrated to effect the acceptance of pandemic influenza vaccine was having seasonal influenza vaccine. It is very important to inform this group having the major risk, to remind and advise the vaccine in routine controls.

\section{NEUROBRUCELLOSIS IN CHILDHOOD: FOUR NEW CASES AND A REVIEW OF THE LITERATURE}

doi:10.1136/archdischild-2012-302724.0840

HA Ayyash. Pediatrics Department, Islamic Hospital, Amman, Jordan

Background and Aims Neurobrucellosis accounts for $<1 \%$ of cases of brucellosis in children and has a broad range of clinical manifestations. In this report we describe our experience regarding the epidemiological, clinical, laboratory and therapeutic findings in four children with neurobrucellosis.

Methods During the past nine years we treated four children with neurobrucellosis in the pediatric department, of Islamic Hospital Amman, Jordan.

The diagnosis was based on epidemiological evidence of brucellosis, as well as Serum \& cerebrospinal fluid(CSF) serology, quantitative changes in C.S.F and favorable response to treatment. Therapy consisted of combinations of two or three of the following drugs for three months: rifampin, gentamycin, streptomycin and trimethoprim-sulfamethoxazole.

Results The main presenting clinical features included fever, neck stiffness. Neurologic signs appeared during the active phase in two patients and later in ther two patients. The interval from onset of symptoms to diagnosis was from 3 days to 5 months. The mean age of children was 7.2 years, and the male: female ratio $3: 1$.

Brucella anti bodies were detected in all sera with levels $\geq 320$ in two cases and $\geq 1280$ in the other two cases.

Cultures in the blood or C.S.F for brucella were unrevealing in all patients.

Cerebrospinal fluid showed: lymphocytosis (500-2160) $\mu \mathrm{l}$ in all cases, elevated proteins in three cases, decreased glucose in two and a Brucella microaggglutination test titre of of $\geq 1: 80$ in 2 cases.

Treatment was successful in all patients after 12 months.

Conclusion We suggest that neurobrucellosis should be considered when neurological manifestations ensues with unknown etiology in endemic areas.

\section{SALMONELLA TYPHI SEPTICEMIA WITH ACUTE RENAL FAILURE IN A 11 YEAR OLD BOY SECONDARY TO PROLIFERATIVE GLOMERONEPHRITIS: A CASE REPORT}

doi:10.1136/archdischild-2012-302724.0841

GS Dhooria, HS Bains, D Bhat. Pediatrics, Dayanand Medical College \& Hospital, Ludhiana, India
An 11 year-old boy was admitted to our hospital because of high fever, gross hematuria and pain in abdomen. He also had hypertension, nephrotic range proteinuria with renal failure, for which hemodialysis was required. Salmonella Typhi was isolated from blood culture and was diagnosed to have typhoid fever. In view of low C3 levels, renal biopsy was done, showed evidence of proliferative glomerulonephritis. On discharge, he had mildly deranged renal function with persistence of gross hematuria and proteinuria which gradually resolved over a period of one year. Renal involvement with enteric fever is noticed only in $2-3 \%$ cases. The common complications of typhoid related to the urinary tract include cystitis, pyelitis, pyelonephritis, and mild proteinuria. Few cases have been reported of acute nephritic syndrome in typhoid fever requiring renal replacement therapy. Here, we report a case of Salmonella typhi septicemia associated with acute renal failure secondary to proliferative glomeronephritis.requiring renal replacement therapy.

\section{SLAPPED-CHEEK INFECTIONS IN CHILDREN WITH SEVERE COMPLICATIONS IN PRESCHOOL CHILDREN}

doi:10.1136/archdischild-2012-302724.0842

${ }^{1}$ A Bajraktarevic, 'E Selimic, 'M Miokovic, 'S Trhulj Putica, 'L Kumasin, 'A Skopljak, ${ }^{1} \mathrm{~N}$ Dizdarevic Kreso, ${ }^{2} \mathrm{~B}$ Djukic, ${ }^{3} \mathrm{~A}$ Selimovic, ${ }^{3} \mathrm{E}$ Mujicic Selimovic, ${ }^{4} \mathrm{~A}$ Drnda, ${ }^{5} \mathrm{Z}$ Jatic, ${ }^{6}$ Suljevic, ${ }^{7 J}$ Gutic, ${ }^{7} \mathrm{M}$ Ridzal. 'Pediatrics Department, Public Health Institution of Sarajevo Canton; ${ }^{2}$ Pediatrics Department, First Medical Aid; ${ }^{3}$ Pulmonology Department, Pediatrics Clinic; ' ${ }^{4}$ ediatrics Departrment, Infectious Clinic Sarajevo; ${ }^{5}$ Department for Family Medicine, Medical Faculty Sarajevo; ${ }^{6}$ Laboratory Biochemistry Department, Clinical Medical Center; ${ }^{\top}$ Perinatology Department, General Hospital Sarajevo, Sarajevo, Bosnia-Herzegovina

Background The most common illness caused by parvovirus B19 infection is 'fifth disease', a mild rash illness that occurs most often in children. The infection often results in no obvious illness. It commonly infects children and typically causes a mild rash that may resemble a "slapped-cheek". Other symptoms that can occur include joint pain (arthralgia), fever and general flu-like symptoms.

Methods Children who are at risk of severe parvovirus complications might benefit from blood tests that can help determine if they're immune to parvovirus or if they've recently become infected. Most cases of slapped cheek syndrome diagnosed by making a visual examination of the distinctive rash. No further testing was usually required in children during three years period 20092011 in capital town of Bosnia, Sarajevo.

Results Parvovirus infection in children with anemia may stop the production of red blood cells and cause an anemia crisis. Children with severe anemia may need to be hospitalized and receive blood transfusions. Percentage of hospitalized of parvivirus infections in children with anaemia is $19 \%$ in Bosnian preschool children, what is only less $1 \%$ of complications in this disease.

Conclusions Fifth disease can cause fetal anemia, which if undetected can have severe consequences. Several days after the appearance of early symptoms, a distinctive bright red facial rash may appear usually on both cheeks. In most children, parvovirus infection is mild and requires little treatment. Slapped cheek is actually the Parvovirus B19 that only affects humans, especially younger children.

\section{REFRACTORY CASE OF KAWASAKI DISEASE WITH HEMOLYTIC ANEMIA: CORRELATION WITH MYCOPLASMATIC INFECTION}

doi:10.1136/archdischild-2012-302724.0843

M Kousouri, E Roumbani, C Koutsaftiki, A Balaska, K Papantzimas, N Myriokefalitakis. 1st Pediatric Department, Penteli's Children's Hospital, Athens, Greece

Introduction Kawasaki Disease $(\mathrm{KD})$ is a systematic disease, usually affecting children 6months-5 years old. Optimal therapy is 